\title{
Essai d'explication du mécanisme de la marée dans le golfe de Saint-Malo
}

\section{A suggested explanation of the tide pattern in the gulf of St. Malo}

\author{
PAR H. LACOMBE \\ PROFESSEUR D'OCÉaNOGRAPHIE PHYSTQUe AU MUSÉUM, \\ INGÉNIEUR HYDROGRAPHE EN CHEF (H.G.)
}

\begin{abstract}
L'auteur assimile la cóte ouest du Cotentin d̀ un obstacle à la propagation de l'onde-marée provenant de l'Atlantique, de la même façon qu'une jetée s'oppose da la propagation de la houle et fait naître près de son extrémité une agitation relevant de la diffraction.

En se plaçant dans un cas schématique et impliquant un obstacle indéfini dans un sens (cote onest du Cotentin indéfinie vers le Sud et mer indéfinie vers le Nord), l'auteur détermine la répartition des amplitudes et des heures de marée, ainsi que celle des courants, dans une zone qui est l'image grossièrement idéalisée de la région s'étendant du cap de la Hagne aux Héaux-de-Brehat. Bien que le cas théorique étudié soit très schématique et ne respecte pas les conditions aux limites de la côte nord de Bretagne et la côte anglaise, les concordances obtenues permettent de penser cependant que la diffraction joue un róle important dans la zone étudiée.
\end{abstract}

\begin{abstract}
The author considers the west coast of the Cotentin peninsula as an obstacle opposing the tide wave from the Atlantic Occan, in the same way as a breakwater opposes an ordinary sea wave and causes agitation due to diffraction at its end.

By taking a simplified case and assuming an obstacle which extends indefinitely in one direction (west coast of the Cotentin peninsula extending indefinitely to the South and the sea extending in the same way to the North) the author determines the distribution of amplitude and of currents for the different hours of the tide, in an area which is an idealised representation of the area extending from the Hague Cape to Les Héaux-de-Bréhat. Although the case studied theoretically is greatly simplified and does not respect the boundary conditions on the North coast of Brittany and on the English coast, the agreement obtained makes it reasonable to think that diffraction is of importance in the area considered.
\end{abstract}

\section{I. - INTRODUCTION. - Schématisation du problème}

Dans une conférence sur « Les marées de la Manche », faite à l'Institut Océanographique en novembre $1950(1)\left(^{\star}\right)$, nous émettions l'hypothèse que le golfe de Saint-Malo (cf. planches III et suivantes) constituait une zone de raccordement entre l'onde-marée de caractère progressif se propageant dans la Manche au nord du Cotentin et l'onde-marée sensiblement stationnaire existant à l'ouest de cette péninsule.

Nous rappelions cette interprétation dans l'ouvrage «Courants de marée dans la Manche et

(*) Les numéros entre parenthèses renvoient à la bibliographie en fin d'article. sur les côtes françaises de l'Atlantique » (2, p. 110). Or, l'agitation dans une telle zone de raccordement entre un régime progressif et un régime stationnaire relève de la diffraction. Celleci, surtout étudiée pour la houle et à l'abri d'un jetée $(3,4)$, se réfère ici à une onde bien plus longue, l'onde-marée, et à une zone située devant l'obstacle sur lequel une onde très longue se réfléchit, quelque faible que soit sa pente (M. Miche). Pour un bassin de profondeur constante et au voisinage d'un obstacle semi-indéfini dans un sens, l'analyse mathématique a fourni $(5,6,7)$ la solution rigoureuse du problème en des termes relativement maniables, à l'inverse de ce qui se passe pour une brèche dans un 
obstacle, cas dans lequel la solution esl très complexe $(8,9)$, quoique justiciable de machines à calculer. Par aillenrs, aucune solution approchée dérivée de celle que nous avons indiquée (4) ne nous a semblé applicable devant un obstacle limité.

Aussi présentons-nous ici seulement les résultats auxquels conduit l'assimilation de « l'agitation » due à la marée à la diffraction d'une onde au voisinage du « musoir » (Cap de la Hague) d'une « jetée » semi-indéfinie (partie positive de Ox) constituée par la côte occidentale du Cotentin frappée en incidence normale par:une onde se propageant dans un bassin de profondeur constante.
Il y a naturellement très loin de ces conditions idéales à la réalité : le frottement est important (10); les profondeurs sont variées; le domaine est parsemé d'îles et de récifs qui diffusent l'énergie en toutes directions; l'obstacle n'est pas « semi-indéfini »; l'onde réelle n'est pas une onde « libre» car elle subit l'effet de la force de Coriolis, ete. Mais nous ne recherchons qu'une schématisation et d'ailleurs il est toujours permis de faire telles approximations qui rendent abordable le traitement théorique d'une question, quitte à les abandonner si elles conduisent à un flagrant désaccord avec la réalité. Mais toute interprétation est précieuse qui est susceptible d'éclairer le mécanisme d'un phénomène réel.

\section{II. - Agitation par diffraction au voisinage immédiat du musoir d'une jetée semi-indéfinie réfléchissante (fig. 1)}

L'expression du potentiel des vitesses en un point $x, y$ (ou $r, 0$ ) pour l'incidence normale dans le cas d'une onde de période $\mathrm{T}$, de longueur d'onde $\lambda$ se progageant dans un bassin de profondeur constante $h$ est $(6,7)$ :

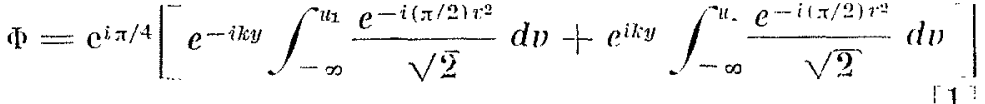

$$
\begin{aligned}
& u_{1}=-\sqrt{\frac{\overline{8} r}{2}} \sin \left(\frac{\pi}{4}-\frac{0}{2}\right) \\
& \int_{2}=-\sqrt{\frac{8 r}{\lambda}} \cos \left(\frac{\pi}{4}-\frac{0}{2}\right)=-\sqrt{\frac{8 r}{\lambda}} \sin \left(\frac{\pi}{4}+\frac{0}{2}\right) \\
& \text { avec: } \\
& k=\frac{2 \pi}{\lambda}
\end{aligned}
$$

On passe à l'amplitude $\eta$ par adjonction du facteur temps $e^{i \sigma t}$ et multiplication de $\Phi$ par $i \sigma / g$ ( $g$ accélération de la pesanteur,

$$
\sigma=(2 \pi / \mathrm{T}) ;(\sigma / k)=c=\sqrt{g \mathrm{H}}) .
$$

Le premier terme en $e^{-i k y}$ de l'expression de $\Phi$ correspond à l'onde incidente, se propageant dans la direction des y positifs, l'autre à l'onde rénéchie.

Comme :

$\int_{-\infty}^{+\infty} \frac{e^{-i(\pi / 2) v^{2}}}{\sqrt{2}} d v=2 \int_{0}^{+\infty} \frac{e^{-i(\pi / 2) \eta^{2}}}{\sqrt{2}} d v=e^{-i(\pi / 4)}$

le mouvement existant devant la jetée $(0=2 \pi)$ pour $x$ très grand positif et $y=0$, c'est-à-dire $u_{1}=u_{2}$ très grands positifs, vaut deux fois l'amplitude incidente et on rencontre donc l'onde stationnaire $e^{-i k y}+e+i k y$.
Au contraire, pour $x$ très grand négatif, $u_{2}$ est infini négatif et l'intégrale correspondante est

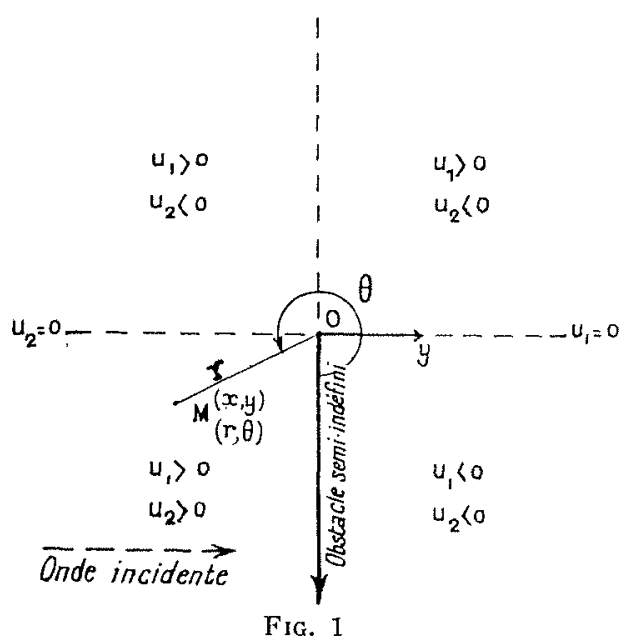

nulle. Il demeure seulement l'onde incidente $e^{-i k y}$ car $u_{1}$ est infini positif. 
L'amplitude réelle du mouvement se trouve aisément en prenant dans le plan de la spirale de Cornu réduite dans le rapport $1, \sqrt{2}$ les deux vecteurs représentant :

$e^{-i k_{y}} \int_{-\infty}^{u_{1}} \frac{e^{-i(\pi / 2) t^{2}}}{\sqrt{2}} d v$ el $\quad e^{i k y} \int_{-\infty}^{u_{2}} \frac{e^{-i(\pi / 2) r^{2}}}{\sqrt{2}} d v$

Le prenier est obtenu en faisant tourner d'un angle $-(2 \pi y / \lambda)=-k y$, le vecteur joignant le point $\Omega^{\prime}$ de paramètre — $\infty$ au point de paramètre $u_{1}$ de la spirale $(y$ est $<0$ ).

Le deuxième en faisant tourner le vecteur

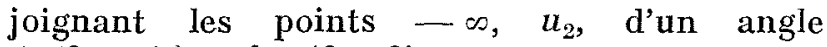
$+(2 \pi y / \lambda)=k y$ (fig. 2).

La somme géométrione des deux vecteurs obtenus donne l'amplitude. Si on le fait tourner de $+\pi / 4$, l'angle avec $O x$ (fig. 2) donne la phase de l'heure de la pleine mer par rapport à l'heure de la pleine mer au point $O$ (1 degré d'angle $=2$ minutes de temps) : en effet, en ce point, où $y=0$ et $u_{1}=u_{2}=0$, l'amplilude est
La transposition à la réalité suppose un choix de la valeur de la longueur d'onde. La distance La Hague-Granville vaut sensiblement $100 \mathrm{~km}$ et, pour des fonds de l'ordre de 25 à $30 \mathrm{~m}$, qui est une moyenne adoptable dans le secteur - ou plutôt une moyenne que nous avons adoptée la longueur d'onde de la marée semi-diurne de période 12 heures est de l'ordre de $700 \mathrm{~km}$. Cette valeur ronde correspond en fait à une profondeur du bassin supposée constante donnée par :

$$
\begin{gathered}
c=\frac{\lambda}{\mathbf{T}}=\frac{700.000}{43.200}=16,2 \mathrm{~m} / \mathrm{s}=\sqrt{g \mathrm{H}}, \\
\text { d'où } \mathrm{H}=26,8 \mathrm{~m}
\end{gathered}
$$

et la distance La Hague-Granville vaut sensiblement $7 / 7$.

Pour $y=0$, soit sur le rivage ouest du Cotentin, les deux vecteurs donnant $\Phi$ sont en phase et $u_{1}=u_{2}=\sqrt{4} r / \lambda$. On a alors très aisément

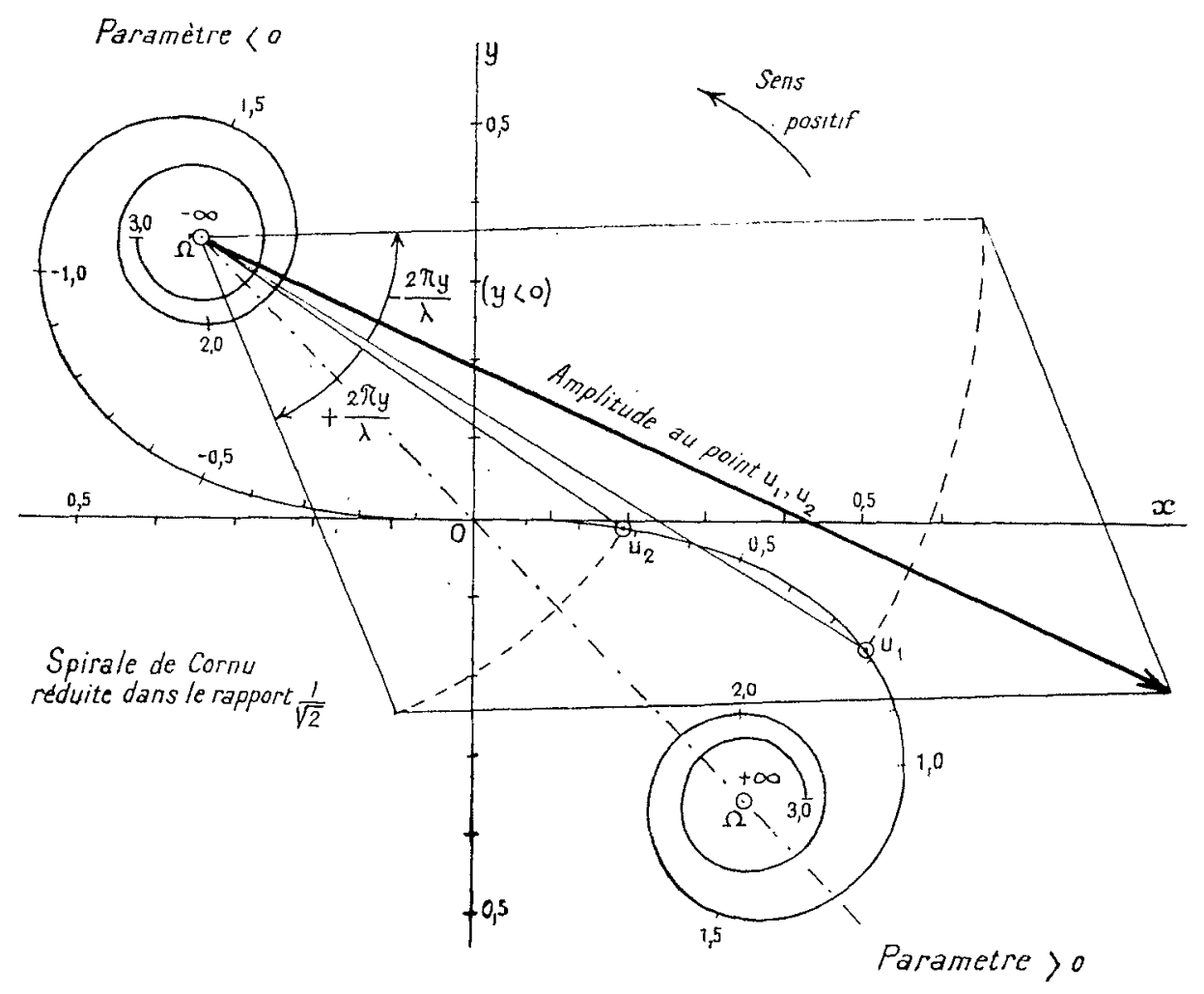

Fia. 2

donnée par deux fois la distance $\Omega^{\prime} O$, soit $\Omega^{\prime} \Omega$ (fig. 2), c'est-à-dire est égale à l'unité; et si on lait tourner $\Omega^{\prime} \Omega$ de $+\pi / 4$, ce vecteur coïncide avec $\mathrm{O} x$ (fig. 2 ); ce sera notre origine des phases qui coincide donc avec l'heure de la pleine mer en $O$ (fig. 1), image du cap de La Hague oì l'amplitude vraie peut être prise égale à $3 \mathrm{~m}$ et le marnage $6 \mathrm{~m}$. amplitude et heure de pleine mer. Au voisinage du « musoir 》 de La Hague, $u_{1}=u_{2}$ sont roisins de $O$ et positifs. On montre aisément que la variation d'amplitude au sud de La Hague présente une branche parabolique et est donc très rapide.

Pour $0=\pi, u_{1}$ et $u_{2}$ sont opposés : l'amplitude vaut 1 partout et l'heure de la pleine mer est 
partout celle de La Hague. Il y a donc à La Hague discontinuité de l'amplitude (et des courants théoriquement infinis).

La figure 3 reproduit les lignes calculées d'égale amplitude relative et d'égale heure de pleine mer interpolées à partir des valeurs calculées pour diverses droites issues de La Hague.

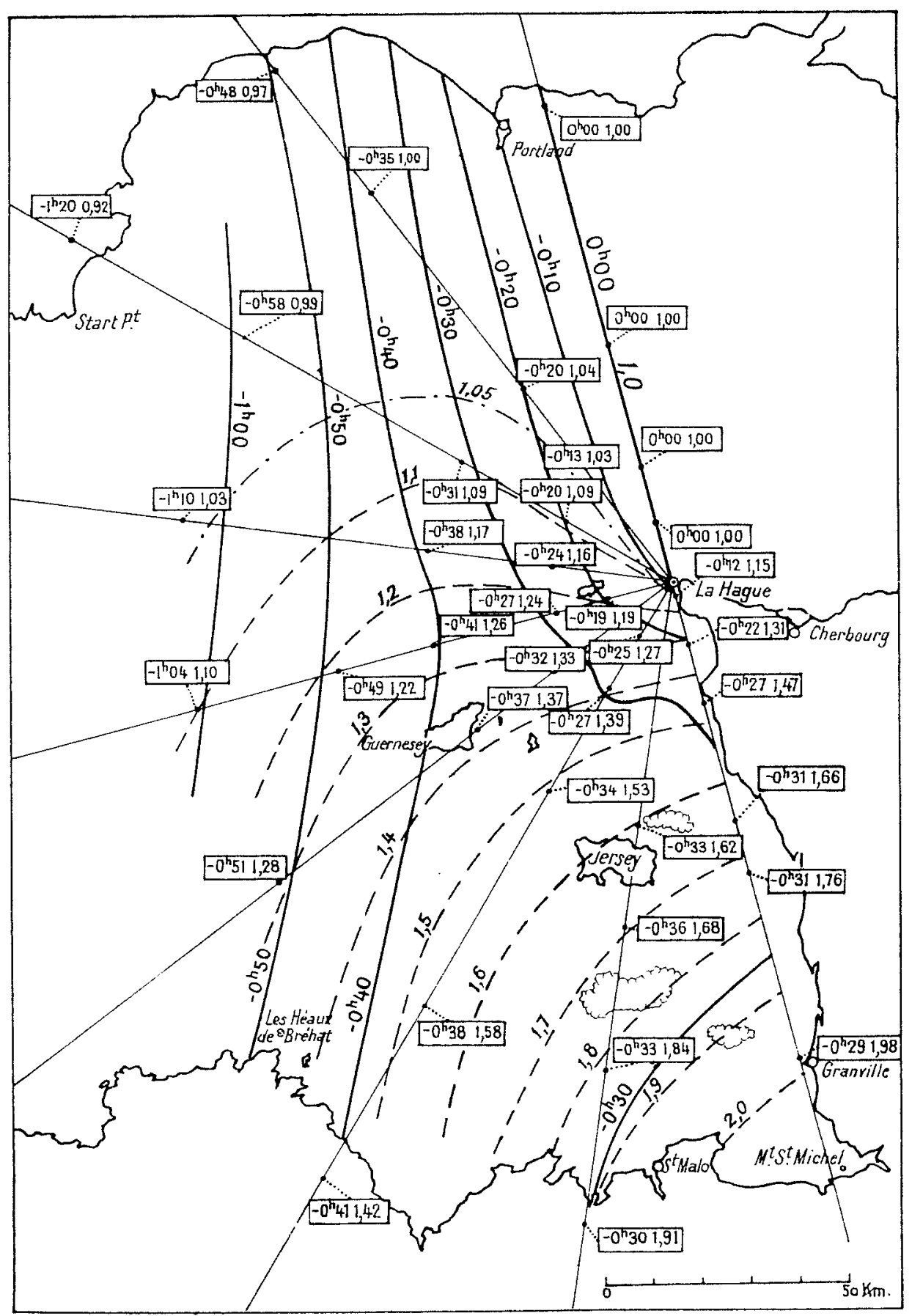

Fig. 3

Marée calculée. Heures et amplitudes rapportées à La Hague. 
Les lignes réelles figurent sur la figure 4 . On voit que la variation d'amplitude calculée sur le rivage et même dans tout le golfe correspond assez bien à la réalité, et qu'il existe bien, dans ce régime, comme dans la réalité, une vaste plage où l'heure de la pleine mer se produit presque atl même instant, l'instant théorique étant postérieur de 25 minutes à 30 minutes à la réalité (35 minutes au lieu de 1 heure environ).

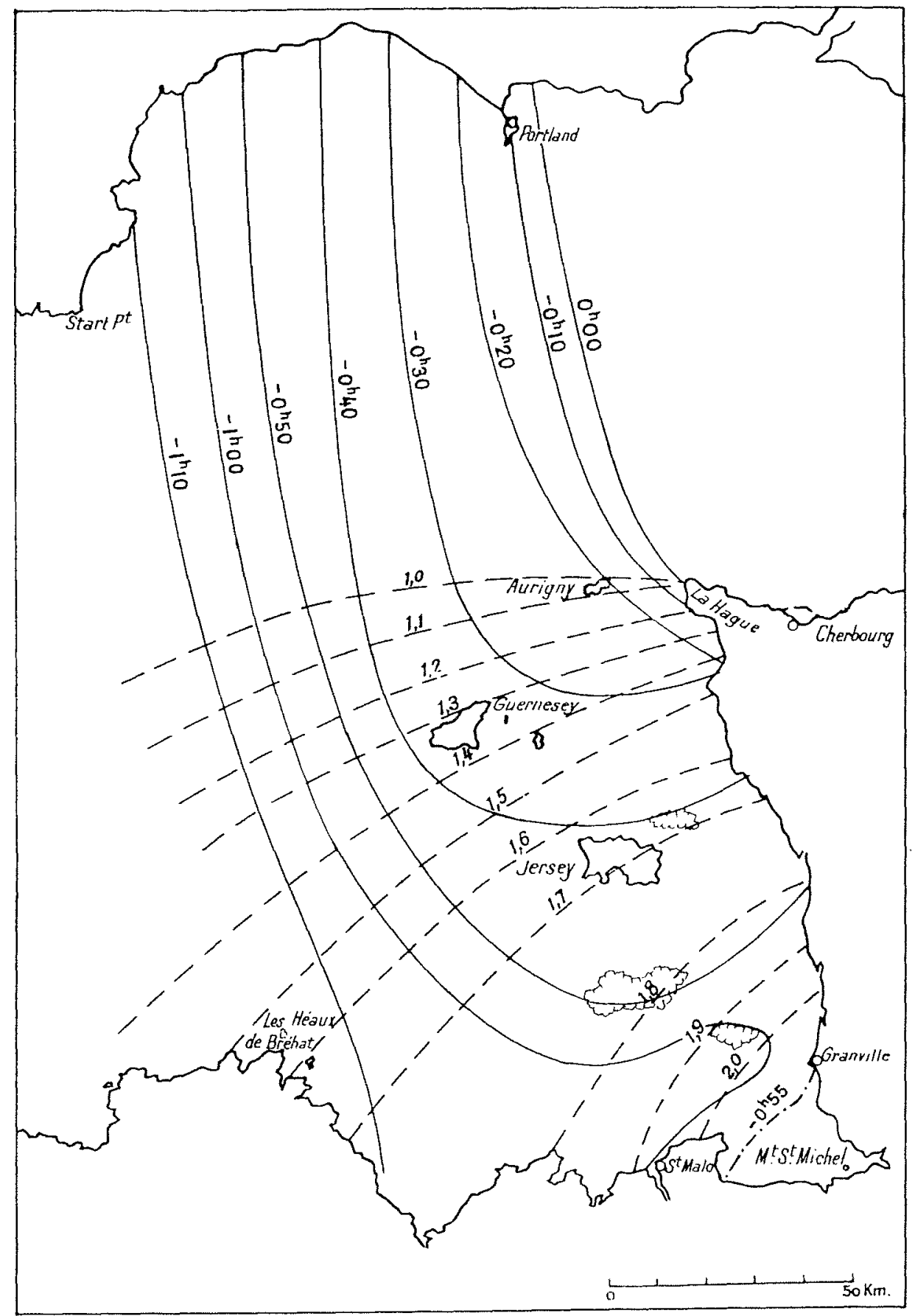

FIG. 4

Marée réelle. Heures et amplitudes rapportées à La Hague. 


\section{III. - Etude des courants}

Une confrontation supplémentaire avec la réalité peut être obtenue par le calcul des courants théoriques.

La dérivation de $\Phi$ donne les vitesses :

$$
\mathrm{U}=-\frac{\partial \omega}{\partial x}, \quad \mathrm{~V}=-\frac{\partial \omega}{\partial y}
$$

Elant donné qu'on compte plus normalement
U positif vers le nord, nous avons calculé les expressions de $-U$ et de $V$. Mais il faut noter que dans l'explication précédente nous avons confondu $\Phi$ et l'amplitude $\eta$.

Or, on a :

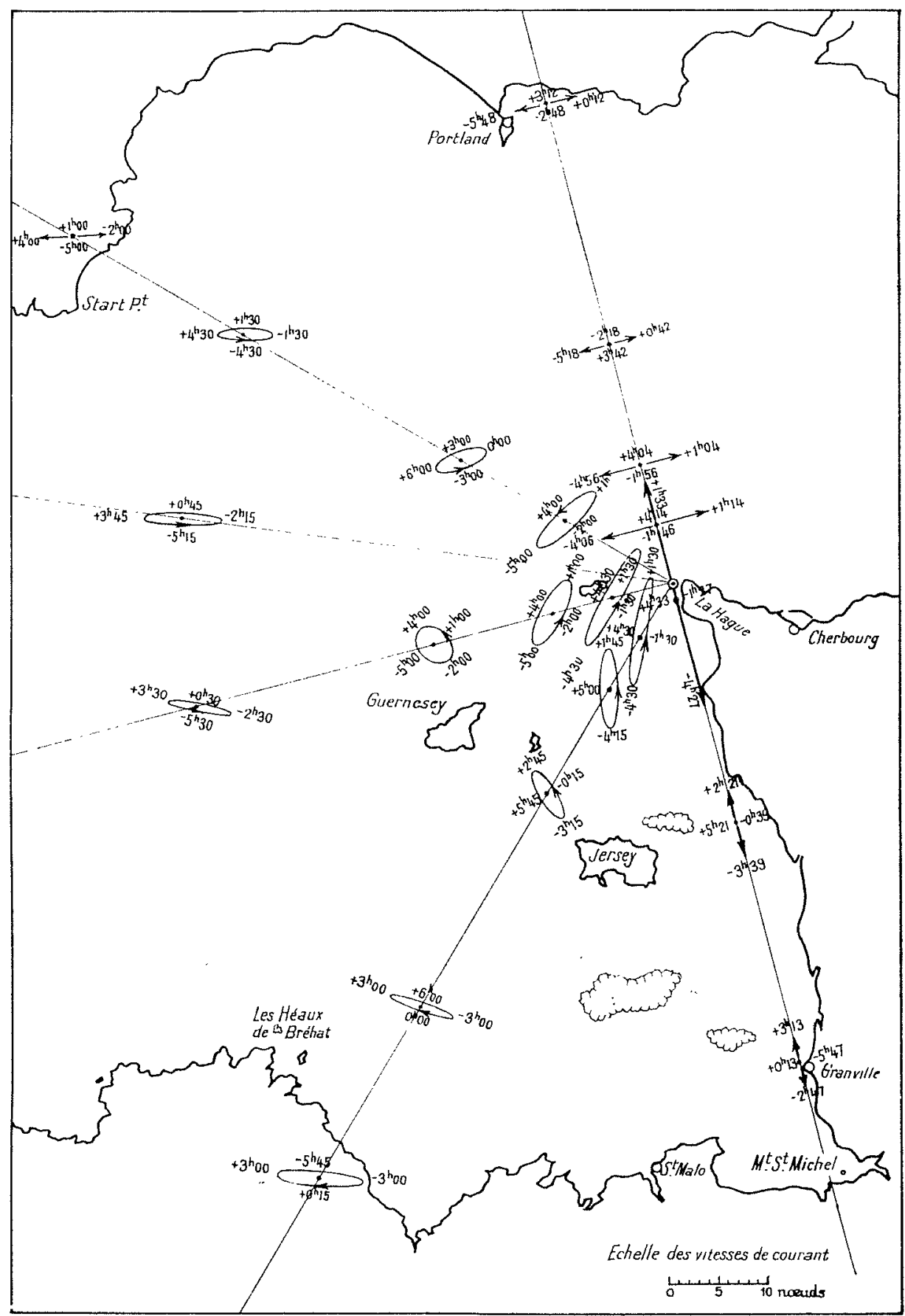

FIG. 5

Courants calculés pour une amplitude totale de $6 \mathrm{~m}$ à La Hague. Heures rapportées à la pleine mer à La Hague. 
Comme on a pris comme référence de $\Phi$ en heure et grandeur la valeur et heure de $\eta$ à La Hague, nous devons, pour avoir les vitesses, diviser la valeur de $\Phi$ par $i \sigma / g$, soit la multiplier par - $i g / \sigma$, on a alors le courant afférant à l'amplilude 1 à La Hague. Formant alors :

$$
\frac{\partial \eta}{\partial x}=+\frac{i g}{\sigma} \quad \frac{\partial \Phi}{\partial x}=-\mathrm{U}
$$

et :

$$
\frac{\partial \eta}{\partial y}=-\frac{i g}{\sigma} \quad \frac{\partial \Phi}{\partial \eta}=\mathrm{V}
$$

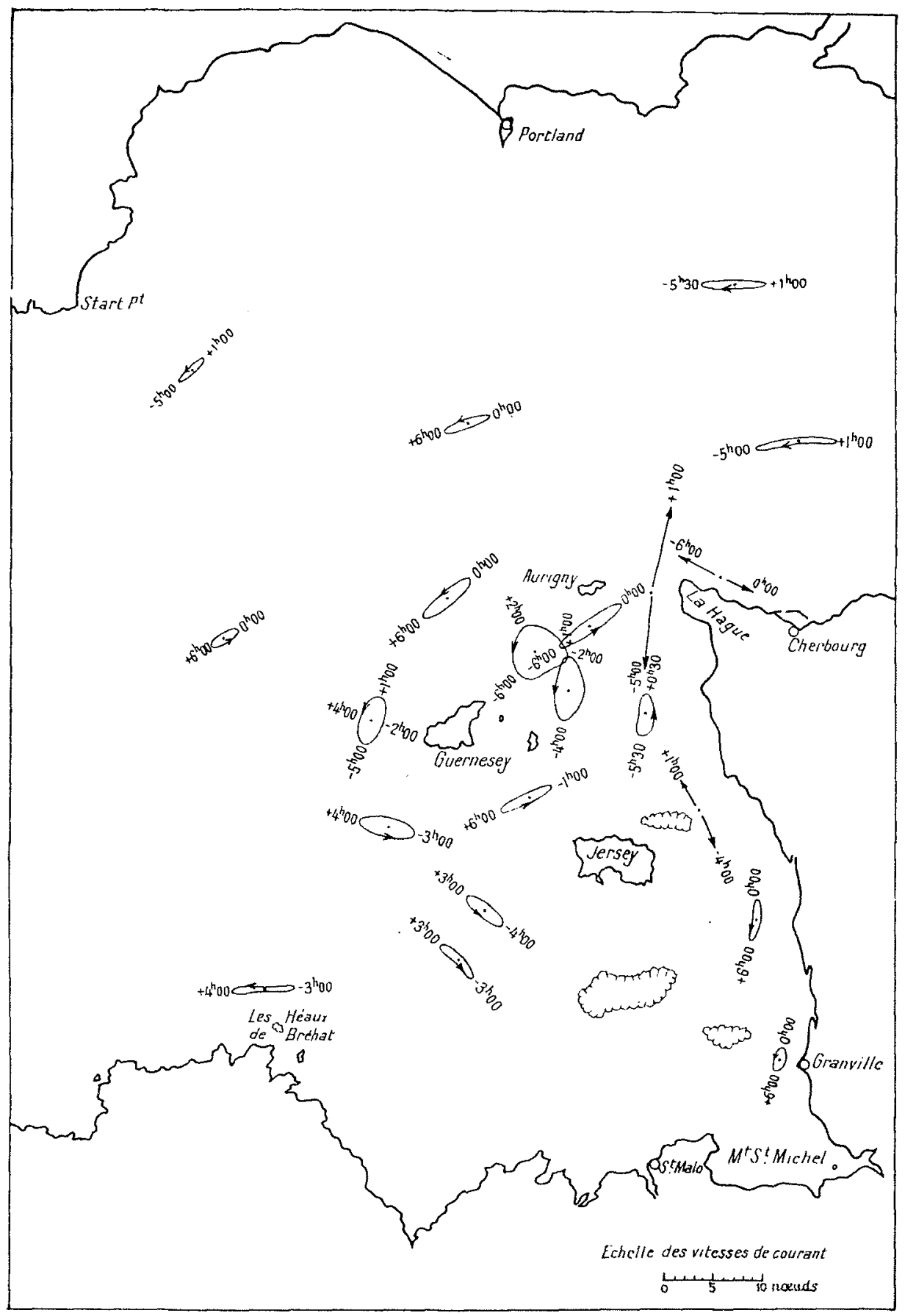

FIG. 6

Courants réels pour une amplitude totale de $6 \mathrm{~m}$ à La Hague. Heures rapportées à la pleine mer à La Hague. 
on obtient (pour $1 \mathrm{~m}$ d'amplitude à La Hague) :

$$
[3]\left\{\begin{array}{l}
\mathrm{V}=\frac{g}{c} \mathrm{e}^{i \pi / 4}\left[e^{-i k y} \int_{-\infty}^{u_{1}} \frac{e^{-(i \pi / 2) v^{2}}}{\sqrt{2}} d v-\cdots e^{i k y} \int_{-\infty}^{u_{2}} \frac{e^{-(i \pi / 2) v^{2}}}{\sqrt{2}} d v-\frac{i}{4 \pi \sqrt{2}} \frac{\lambda}{r} e^{-2 i \pi r / \lambda}\left(u_{1}-u_{2}\right)\right. \\
=\frac{g}{c} e^{i \pi / t}\left[e^{-i k y} \int_{-\infty}^{u_{1}} \frac{e^{-(i \pi / 2) v^{2}}}{\sqrt{2}} d v-e^{i k^{\prime} y} \int_{-\infty}^{u_{2}} \frac{e^{-(i \pi / 2) t^{2}}}{\sqrt{2}} d v-\frac{i \sqrt{2}}{2 \pi} \sqrt{\frac{\lambda}{r}} \sin \frac{0}{2} e^{-2 i \pi r \lambda}\right]
\end{array}\right.
$$

par mètre d'amplitude à La Hague. L'amplitude réelle en vive-eau moyenne est de $3 \mathrm{~m}$ : il y a donc environ $6 \mathrm{~m}$ de « marnage »,.

On voit que $V=0$ quand $u_{1}=u_{2}$, soit $\theta=0$ ou $2 \pi$, c'est-à-dire devant la jetée. U est nul sur la partie négative de $o x\left(u_{1}=-u_{2}\right)$ et pour $x$ très grand négatif on a $\mathrm{V}=(g / c)=\sqrt{g / \mathrm{H}}$ par mètre d'amplitude : l'onde est progressive pure.

Les conditions aux limites ne sont pas vérifiées dans la région de Granville et de la côte nord de Bretagne où la composante nord-sud du courant n'est pas nulle comme elle le devrait. On trouve devant Granville une vitesse théorique de 2 nouds. De même sur la côte anglaise, au $\mathrm{N} W$ de La Hague, aux environs de ce qui serait Start Point, on a un courant nord-sud de 0,6 noud. Les conditions aux limites ne sont donc pas respectées.

Les figures 5 et 6 présentent les roses de courants théoriques et les roses réelles. Ces roses sont obtenues en portant à chaque instant le vecteur vitesse à partir du point où la vitesse est calculée ou mesurée. On voit que dans l'ensemble la giration en sens inverse des aiguilles d'une montre, qui est une caractéristique essentielle des courants réels dans cette zone, ressort nettement, de même que l'augmentation de courant, vers le raz Blanchard. Les heures des renverses et l'allure des heures calculées suivent d'assez près la réalité aux abords de La Hague dans un rayon d'une cinquantaine de $\mathrm{km}$. On a également une concordance assez frappante au S W de la ligne entre Jersey et les Héaux de Bréhat. Mais dans le $\mathrm{N} W$, il y a une discordance dans les heures qu'on peut fort légitimement attribuer au caractère d'onde stationnaire partielle qu'à la marée réelle, en raison de la réflexion sur la côte de la Somme au Cap Gris$\mathrm{Nez}$ et qui provoque une concordance des phases des courants réels dans une vaste zone de part et d'autre de La Hague [Voir (2) page 31].

\section{Conclusion}

Il semble que la concordance approximative des amplitudes, et dans une certaine mesure celle des courants, notamment pour leur sens de rotation, permet d'affirmer que, pour une part appréciable, le mécanisme de la marée dans le golfe de Saint-Malo peut s'expliquer par la diff raction. Il semble que des essais sur modèle seraient sus- ceptibles de mieux éclairer le problème et, en particulier, permettraient de dire quelle peut être la part de la diffraction et, par différence avec la réalité, celle de la force de Coriolis, que l'on pourrait peut-être apprécier sur un petit bassin très schématique mais tournant. 